\title{
Identified Serotonergic Neurons LCB1 and RCB1 in the Cerebral Ganglia of Aplysia Produce Presynaptic Facilitation of Siphon Sensory Neurons
}

\author{
Steven L. Mackey,' Eric R. Kandel, ${ }^{1,2,3}$ and Robert D. Hawkins ${ }^{1,2}$ \\ 'Center for Neurobiology and Behavior, College of Physicians and Surgeons, Columbia University, ${ }^{2}$ New York State \\ Psychiatric Institute, and ${ }^{3}$ Howard Hughes Medical Institute, New York, New York 10032
}

\begin{abstract}
Several lines of evidence suggest that 5-HT plays a significant role in presynaptic facilitation of the siphon sensory cells contributing to dishabituation and sensitization of the gill- and siphon-withdrawal reflex in Aplysia. Most recently, Glanzman et al. (1989) found that treatment with the 5-HT neurotoxin, 5,7-DHT markedly reduced both synaptic facilitation and behavioral dishabituation. To provide more direct evidence for a role of 5-HT, we have attempted to identify individual serotonergic facilitator neurons. Hawkins (1989) used histological techniques to locate several serotonergic neurons in the ring ganglia that send axons to the abdominal ganglion and are therefore possible serotonergic facilitators. These include one neuron in the B cluster of each cerebral ganglion, which we have identified electrophysiologically and named the CB1 cells. Both glyoxylic acid histofluorescence and 5-HT immunofluorescence indicate that the CB1 neurons are serotonergic. In a semiintact preparation, the CB1 neurons respond to cutaneous stimulation which produces dishabituation and sensitization (such as tail shock) with an increase in firing, which may outlast the stimulation by 15 min. Intracellular stimulation of a CB1 neuron in a manner similar to its response to tail shock produces facilitation of the EPSPs from siphon sensory neurons to motor neurons, as well as broadening of the action potential in the sensory neurons in tetraethylammonium solution. These results strongly suggest that the identified serotonergic CB1 neurons participate in mediating presynaptic facilitation contributing to dishabituation and sensitization of the gill- and siphon-withdrawal reflex in Aplysia.
\end{abstract}

As reviewed in the 2 preceding papers (Glanzman et al., 1989; Hawkins, 1989), several lines of evidence suggest that 5-HT plays a significant role in presynaptic facilitation of the siphon sensory cells contributing to dishabituation and sensitization of the gill- and siphon-withdrawal reflex in Aplysia. Application of serotonin mimics the effects of cutaneous or nerve stimulation in producing facilitation of the EPSPs from sensory cells to

Received Feb. 27, 1989; revised June 6, 1989; accepted June 12, 1989.

This work was supported by grants from the National Institute of Health (MH26212) and the Howard Hughes Medical Institute. We thank D. Glanzman, L. Eliot, and I. Kupfermann for their comments, K. Hilten and L. Katz for preparing the figures, and H. Ayers and A. Krawetz for typing the manuscript.

Correspondence should be addressed to Dr. Robert D. Hawkins, Center for Neurobiology and Behavior, College of Physicians and Surgeons, Columbia University, 722 West 168 th Street, New York, NY 10032.

Copyright (C) 1989 Society for Neuroscience $0270-6474 / 89 / 124227-09 \$ 02.00 / 0$ follower cells, as well as broadening of action potentials and elevation of cAMP in the sensory neurons (Brunelli et al., 1976; Klein and Kandel, 1978; Bernier et al., 1982). Furthermore, 5-HT is detectable in the abdominal ganglion (the location of the sensory cells) by several methods, and 5-HT immunoreactive processes come in close contact with the sensory cells (Kistler et al., 1985). Most recently, we have found that treatment with the 5-HT neurotoxin, 5,7-DHT, significantly reduced both facilitation of the sensory cells and dishabituation of the reflex (Glanzman et al., 1989).

However, since this evidence is all indirect, and since at least 2 other transmitters (the small cardioactive peptide SCP and the unknown transmitter of the L29 cells) can produce facilitation of the sensory cells (Hawkins et al., 1981; Abrams et al., 1984), we sought more direct evidence for a role of 5-HT in facilitation, dishabituation, and sensitization. The preceding paper (Hawkins, 1989) describes experiments combining glyoxylic histofluorescence with retrograde fluorescent labeling, which located approximately 10 serotonergic neurons in the ring ganglia that send axons to the abdominal ganglion and are therefore possible serotonergic facilitator neurons. These include 4 neurons in each pedal ganglion and 1 neuron in the B-cell cluster of each cerebral ganglion. Since the cerebral neurons showed the most consistent double labeling, we have studied them first. In this paper, we show that these identified serotonergic cells are excited by cutaneous stimuli that produce dishabituation and sensitization, and that firing them produces presynaptic facilitation of the siphon sensory cells.

\section{Methods}

Electrophysiology. Aplysia californica weighing 40-250 gm were obtained from Pacific Biomarine (Venice, CA) or Marinus (Long Beach, CA). Animals were anesthetized by injection of isotonic $\mathrm{MgCl}_{2}(25-$ $40 \%$ body weight) and were dissected in a $50 \%$ isotonic $\mathrm{MgCl}_{2}, 50 \%$ artificial seawater solution. When studying neural responses to cutaneous stimulation, we used a modified "split-foot" preparation (Hening et al., 1979). The viscera, buccal mass, opaline gland, and purple gland were excised, and the anterior half of the foot and back were bisected longitudinally, revealing the nervous system and blood vessels. The preparation was then pinned dorsal side up to the wax floor of a lucite recording chamber filled with circulating, aerated artificial seawater. The cut end of the cephalic artery was cannulated and perfused with artificial seawater (in mM: $460 \mathrm{NaCl}, 10 \mathrm{KCl}, 55 \mathrm{MgCl}_{2}, 11 \mathrm{CaCl}_{2}, 10 \mathrm{HEPES}$, $\mathrm{pH}$ 7.6) at room temperature. The cerebral ganglia were pinned on a Sylgard stage and partially desheathed, and the pleural-abdominal connectives were wrapped around stimulation posts to facilitate identification of neurons. Neurons were impaled with single-barreled glass microelectrodes filled with $2.5 \mathrm{M} \mathrm{KCl}$ for recording and stimulation through a Wheatstone bridge circuit. Cells were examined for their response to cutaneous tactile stimulation using a glass probe or electrical 
stimulation (75 mA a.c., $1.0 \mathrm{sec}$ duration) using capillary electrodes. The cells were then filled with rhodamine and processed for glyoxylic histofluorescence as described in the preceding paper (Hawkins, 1989).

When examining facilitation of siphon sensory neuron-motor neuron synapses, we discarded the body except for the tail, which was left connected to the ring ganglia via the posterior pedal nerves (P9). The ring and abdominal ganglia were dipped in $0.5 \%$ glutaraldehyde for $\mathbf{4 5}$ sec, pinned to the Sylgard floor of the recording chamber, and partially desheathed. The preparation was then perfused with artificial seawater at room temperture. Candidate cerebral cells, siphon sensory neurons (LE cells), and siphon or gill motor neurons (usually LFS or L7) were impaled. The motor neuron was hyperpolarized $30 \mathrm{mV}$ below spike threshold and tested for receiving an EPSP from LE cells. EPSPs were considered monosynaptic if their latency was less than $10 \mathrm{msec}$ and constant. If the PSPs were complex, the amplitude was measured at the first peak or inflection point.

When examining spike broadening in the siphon sensory cells, we used the isolated ring and abdominal ganglion preparation. Cerebral cells and LE sensory cells were impaled and the perfusion was changed to $100 \mathrm{~mm}$ tetraethylammonium chloride (TEA) in artificial seawater. Five-millisecond depolarizing current pulses were injected into the sensory cells causing them to fire single action potentials. The duration of the action potential was measured at the midpoint of its vertical excursion.

Immunohistochemistry. In some of the electrophysiology experiments, cerebral cells were filled with Lucifer yellow and processed for serotonin immunoreactivity, using a modification of the whole-mount technique of Longley and Longley (1986). At the end of the experiment, the cells were repenetrated with electrodes filled with $5 \%$ Lucifer yellow in distilled water and filled iontophoretically (500 msec, $1-2 \mathrm{nA}$ hyperpolarizing pulses at $1 \mathrm{~Hz}$ for $15-30 \mathrm{~min}$ ). After iontophoresis, the ring ganglia were fixed using $4 \%$ paraformaldehyde in $0.1 \mathrm{M} \mathrm{Na}$ phosphate (pH 7.4) containing $30 \%$ sucrose at $4^{\circ} \mathrm{C}$ for $24 \mathrm{hr}$. The tissue was then permeablized using Triton X-100 in PBS $(0.1 \mathrm{~m}$ Na phosphate, $0.17 \% \mathrm{Na}$ azide, $2 \%$ Triton X-100) for $24 \mathrm{hr}$. Afterward, the tissue was incubated for $24 \mathrm{hr}$ in polyclonal rabbit anti-5-HT antibody (ImmunoNuclear Corporation, Stillwater, WI) diluted 1:200 in Triton X-100 PBS. Next, the tissue was washed in Triton PBS for another $24 \mathrm{hr}$ and then incubated for $24 \mathrm{hr}$ in rhodamine-labeled goat anti-rabbit antiserum (Cappel Laboratories, Cochranville, PA) diluted 1:50 in Triton PBS. After another 24-hr wash in Triton PBS, the tissue was rinsed in $0.03 \mathrm{M} \mathrm{Na}$ phosphate and mounted in glycerol. The slides were viewed on a Leitz epifluorescence microscope, using the N2 and D or 12 filter packs for rhodamine and Lucifer yellow, respectively, and photographed with Kodak Ektachrome 400 film.

\section{Results}

Hawkins (1989) found that several cells in the B cluster of each cerebral ganglion show 5-HT histofluorescence and several show retrograde fluorescent labeling from the abdominal ganglion, but only one cell in each ganglion shows both. That cell is therefore a potential serotonergic facilitator for the siphon sensory cells. In a series of experiments before we knew how to identify that cerebral cell electrophysiologically (Mackey et al., 1986), we tested whether any cerebral cells that had axons going to the abdominal ganglion (as evidenced by receiving antidromic action potentials from stimulation of the pleural-abdominal connectives) (1) produced facilitation of the EPSP from a siphon sensory neuron to a motor neuron, and (2) showed 5-HT histofluorescence. In those experiments, cerebral cells were stimulated at a relatively high frequency $(10 \mathrm{~Hz})$ for $25-50 \mathrm{sec}$. Ten of thirty-one cerebral B cells with axons in the pleural-abdominal connectives produced facilitation of the sensory neuronmotor neuron EPSP. Only 1 cell in each B cluster produced facilitation; in 2 experiments, such a cell was found in both the left and right clusters of the same animal. At the end of the experiments, some of the cells that produced facilitation were filled with rhodamine and processed for glyoxylic histofluorescence as described in the preceding paper (Hawkins, 1989).

\section{CEREBRAL GANGI ION \\ DORSAL VIEW}

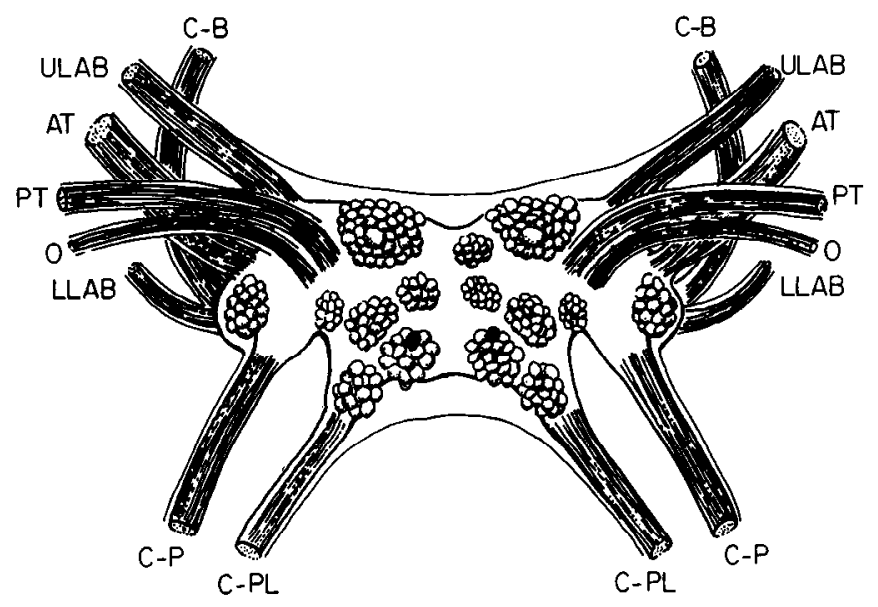

Figure 1. Map of the dorsal surface of the cerebral ganglion showing the approximate locations of the CB1 neurons (filled) in the left and right B clusters. The map of the cell clusters is modificd from JahanParwar and Fredman (1976).

Three of three cells that produced facilitation in 3 different preparations showed positive 5-HT histofluorescence.

The results of these experiments were consistent with the idea that one of the cells in the B cluster of each cerebral ganglion is a serotonergic facilitator. However, to be more confident of that conclusion, it was necessary to identify that cell electrophysiologically. In this paper, we report on identification of an individual neuron in each B cluster (the CB 1 cells) and address the following questions: (1) Are the CB1 cells serotonergic? (2) Are they excited by cutaneous stimuli known to produce dishabituation and sensitization? (3) Does intracellular stimulation of CB1 cells, in a manner similar to their response to cutaneous stimulation, produce facilitation of EPSPs from LE cells to follower cells? (4) Is this effect direct? (5) Is this effect presynaptic?

\section{Electrophysiological identification of $C B 1$ neurons}

We routinely found up to 3 cells in each $B$ cluster that received an antidromic action potential from stimulation of either one or both pleural-abdominal connectives. However, we found that only 1 of those cells also receives excitatory synaptic input from the tail or posterior pedal nerve (P9); the other cells are inhibited. That cell, which we have designated CB1, is pale yellow, approximately $75-100 \mu \mathrm{m}$ in diameter, and is located in the anterior region of the B cluster (Fig. 1). It has a low rate of spontaneous firing, receives spontaneous EPSPs and IPSPs, and receives an antidromic action potential from stimulation of either pleural-abdominal connective.

\section{CB1 neurons show 5-HT histofluorescence and immunoreactivity}

To test whether the CBI cells are serotonergic, we injected them with lissamine rhodamine and processed the ganglion for glyoxylic acid histofluorescence as described in the preceding paper (Hawkins, 1989). Figure $2 A$ shows an identified $C B 1$ cell injected with rhodamine and viewed with rhodamine filters. Figure $2 B$ shows the same cell viewed with histofluorescence filters, revealing positive 5 -HT histofluorescence. As an independent test 


\section{CB 1}

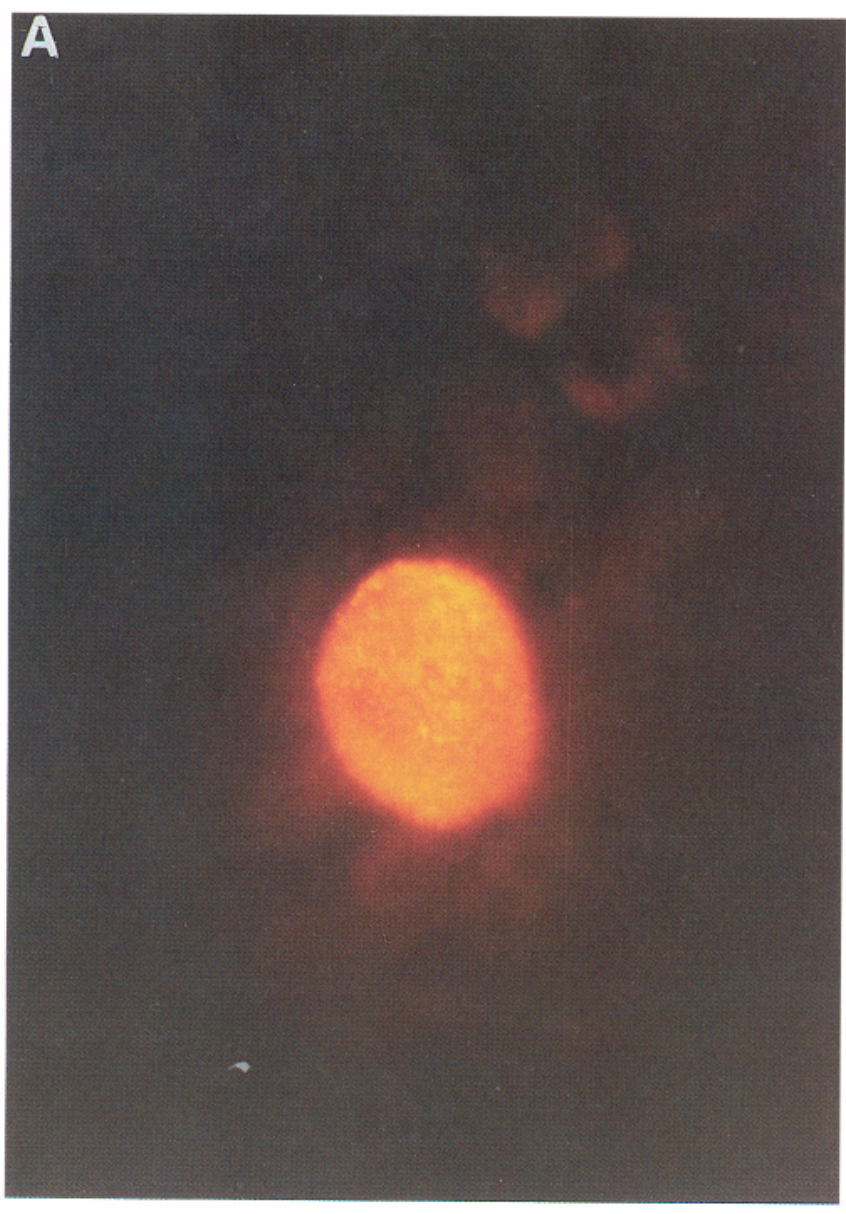

RHODAMINE

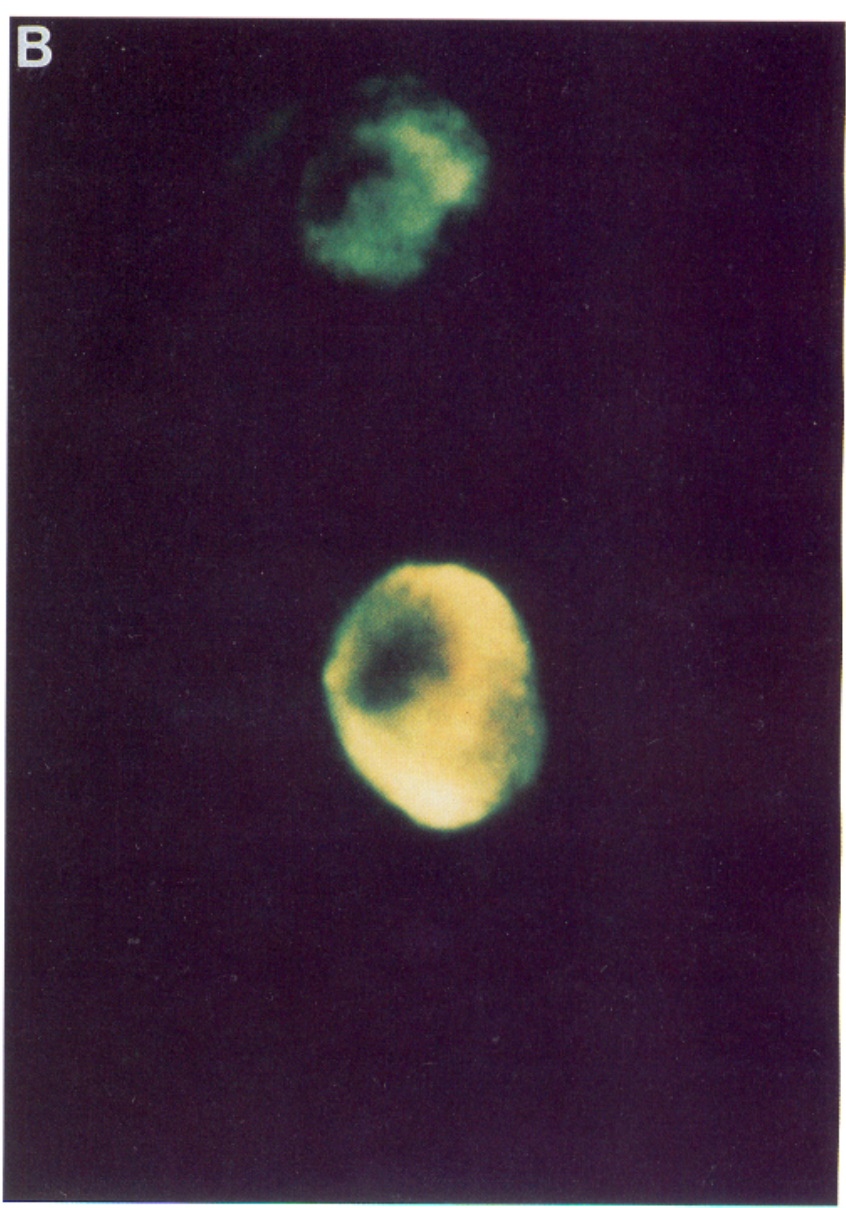

GLYOXYLIC ACID

\section{$100 \mu \mathrm{m}$}

Figure 2. 5-HT histofluorescence in a CB1 neuron. The cell was identified electrophysiologically (see the text) and injected with lissamine rhodamine, and then the ganglion was processed for glyoxylic acid histofluorescence. $A$, The injected cell viewed with rhodamine filters. $B$, The same section viewed with histofluorescence filters, showing serotonin histofluorescence in the CB1 cell.

of whether the CB1 cells are serotonergic, we also examined whether those cells show 5-HT immunoreactivity. Cerebral cells were selected as described above and filled with Lucifer yellow. The cells were then processed for wholemount immunohistochemistry using a polyclonal anti-5-HT antibody. Identified CB1 cells showed positive 5-HT immunoreactivity (Fig. $3 \mathrm{~A}$ ).

These experiments also revealed the axonal branching pattern of CB1: its axon bifurcates and sends one branch to the ipsilateral cerebral-pleural connective, while the other branch crosses the cerebral commissure and enters the contralateral cerebralpleural connective (Fig. $3 B$ ). The positions of the axons in the cerebral-pleural connectives are similar to those of the serotonergic processes ("S1" and "S2") described by Longley and Longley (1986), who showed that those axons project to the abdominal ganglion and branch extensively in the neuropil (Fig. 4). No processes of $\mathrm{CB} 1$ were noted in peripheral cerebral nerves.
CBI neurons are excited by cutaneous stimuli that produce dishabituation and sensitization

We used a semiintact preparation as illustrated in Figure $5 \mathrm{~A}$ to assess the response of cerebral cells to cutaneous stimulation. An example of the response of CB1 neurons is shown in Figure $5 B$ and $C$. CB1 neurons, which show irregular spiking at rest, respond with a phasic increase in firing to moderate tactile stimulation anywhere on the body surface (Fig. $5 B$ ). A noxious stimulus such as tail shock, however, causes a prolonged increase in firing rate, which in some cases lasts over $15 \mathrm{~min}$ (Fig. $5 \mathrm{C}$ ). This response was observed in CB1 cells in several different preparations and could be produced repeatedly in the same preparation. The prolonged response is not due to continued stimulation from pulling against the restraints, since stimulation of the posterior pedal nerve (P9) also produced a similar response 
RIGHT CEREBRAL GANGLION

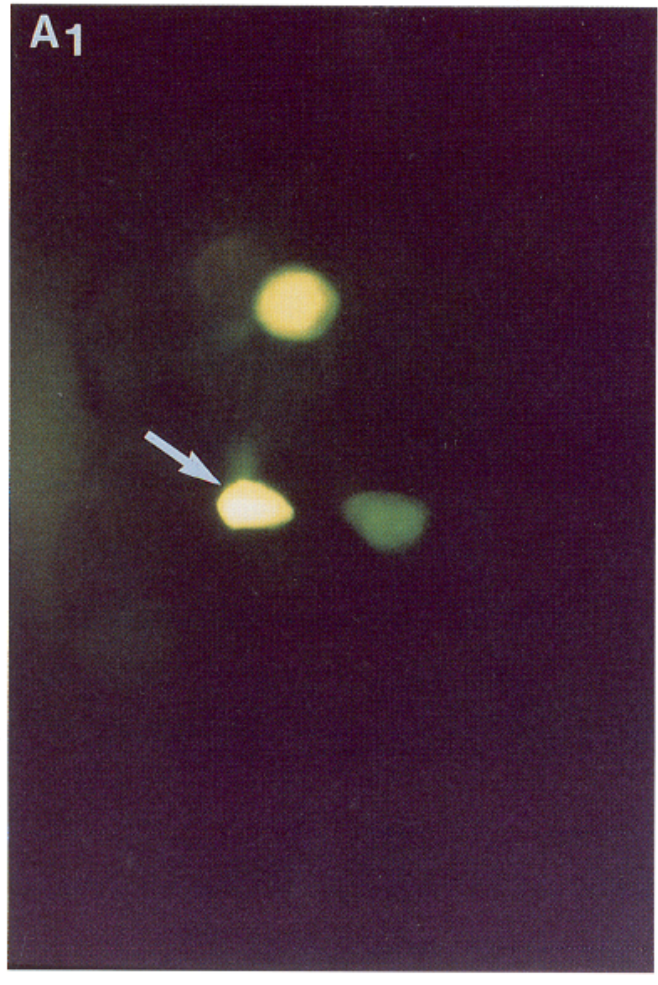

LUCIFER YELLOW

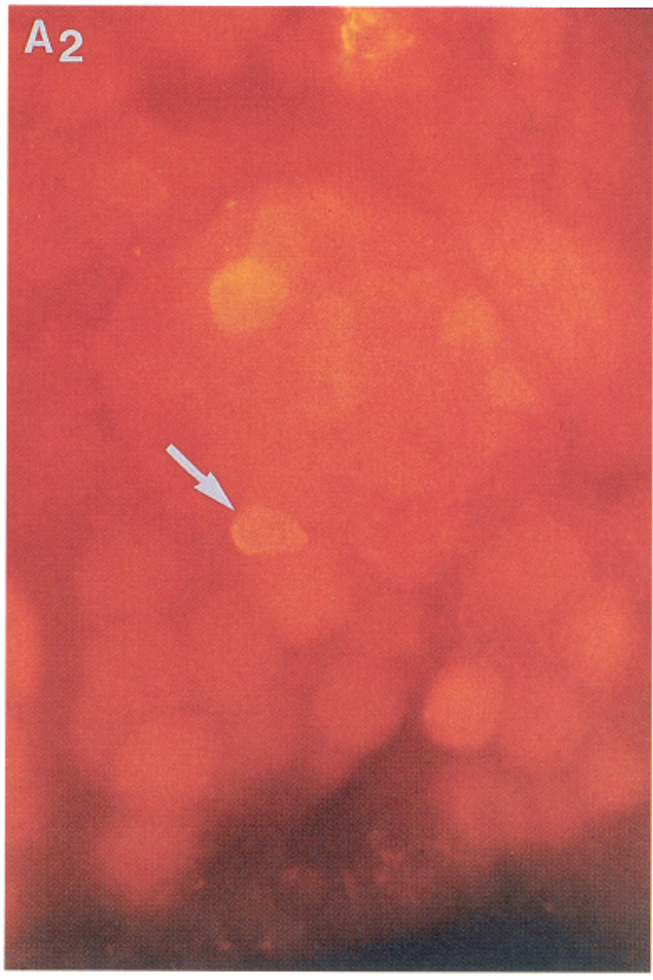

SEROTONIN

LEFT CEREBRAL - PLEURAL CONN.

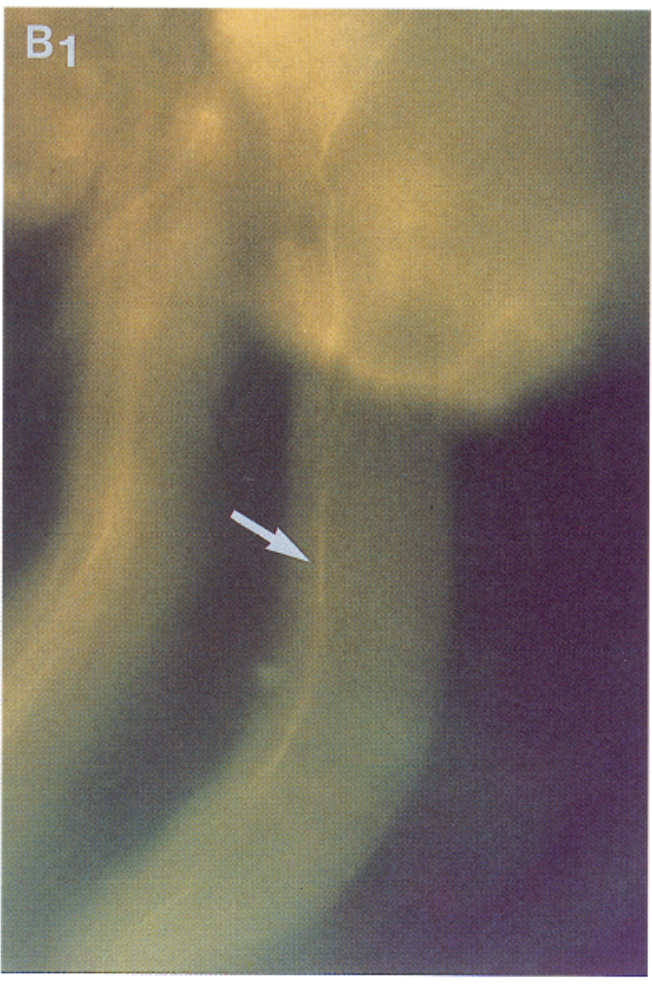

LUCIFER YELLOW

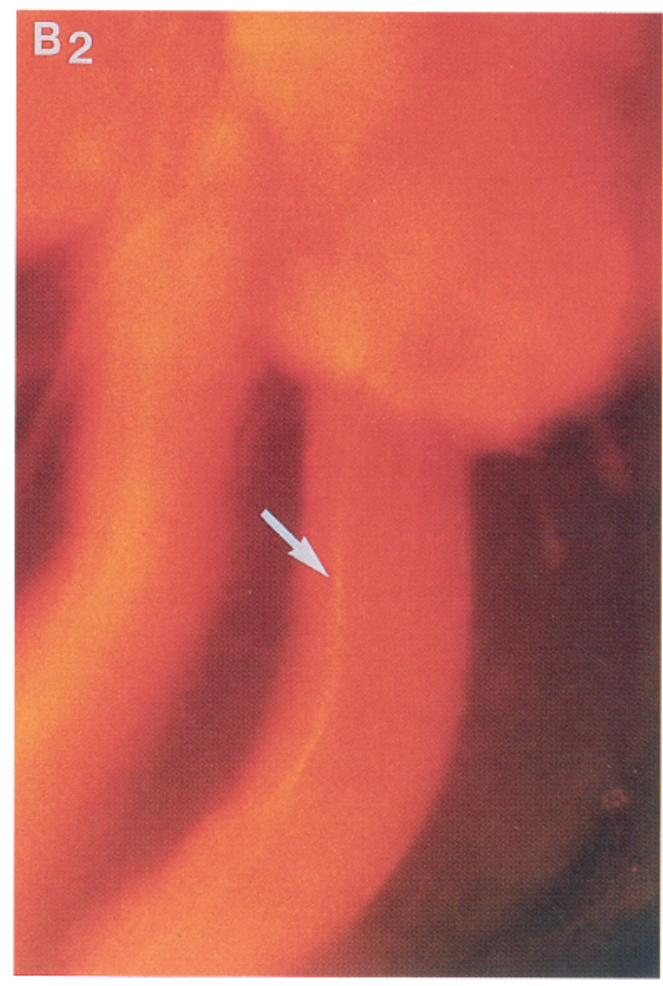

SEROTONIN 
in the isolated nervous system. The cellular basis of this prolonged increase in firing rate has not yet been investigated.

\section{Stimulation of CB1 produces facilitation of EPSPS from $L E$ cells to follower cells}

We next addressed the question of whether stimulating the CB1 cells in a manner similar to their response to tail shock produces reliable facilitation of the EPSP from LE sensory cells to follower cells. CB1 neurons were identified as described above. An LE sensory cell and a follower neuron (usually an LFS motor neuron) were then impaled. Follower neurons were hyperpolarized by $30 \mathrm{mV}$ to prevent spiking. The LE cell was stimulated intracellularly with a brief current pulse causing it to fire an action potential once every $30 \mathrm{sec}$. After at least 3 pretest stimuli, the CB1 cell was fired intracellularly at a rate of $1-3 \mathrm{~Hz}$ for 180 $200 \mathrm{sec}$. The EPSP from the LE cell to the follower cell was tested for 6 trials during CB1 stimulation and 3 trials poststimulation.

An example of the results is shown in Figure $6 \mathrm{~A}$. The average results from 10 experiments are plotted in Figure $6 B$. The amplitude of the EPSP has been normalized to the last pretest $(-1)$. As can be noted, the EPSP decreased in amplitude slightly from pretest -3 to -1 . After the start of CB1 stimulation, the amplitude of the EPSP increased on average to a peak of $142 \pm$ $17 \%$ of pretest by trial 3 , and facilitation was maintained throughout CB1 stimulation. After the end of CB1 stimulation, the EPSP declined. At posttest 1, the EPSP was on average 128 $\pm 10 \%$ of pretest; by posttest 3 , the EPSP was $107 \pm 8 \%$ of pretest. The average amplitude of the EPSP during and following CB1 stimulation was significantly greater than pretest $\left(F_{1,72}=\right.$ $45.6, p<0.01$ ), and planned comparisons at the individual time points show significant facilitation on trials 3,4 , and 6 during CB1 stimulation and posttest 1 ( $p<0.01$ in each case). The average magnitude of facilitation, although modest, is similar to that produced by tail stimulation (Mackey et al., 1987). Although the duration of facilitation is substantially less than that seen after tail shock, CB1 was also stimulated for a shorter interval than it fires after tail shock (cf. Fig. $6 \mathrm{~A}$ and Fig. $5 \mathrm{C}$ ). This suggests that at least part of the time course of facilitation following tail shock may be due to continued firing of the CB1 neurons and continued release of 5-HT.

These results support the idea that the $\mathrm{CB} 1$ cells mediate some of the facilitatory effects of tail shock. Tail shock is still capable of producing facilitation when the cell body of an individual CB1 neuron is hyperpolarized to prevent it from firing, suggesting that a single CB1 cell does not mediate all of the facilitatory effects of the shock (although this procedure may not prevent firing in distant processes of the cell). We have not yet performed enough to these experiments to know whether there is a quantitative reduction in the facilitation, nor have we hyperpolarized both $\mathrm{CB} 1$ cells simultaneously.

In 5 of the 10 experiments illustrated in Figure $6 B$, EPSP facilitation during and following CB1 stimulation was tested in an artificial seawater solution with elevated divalent cations (in

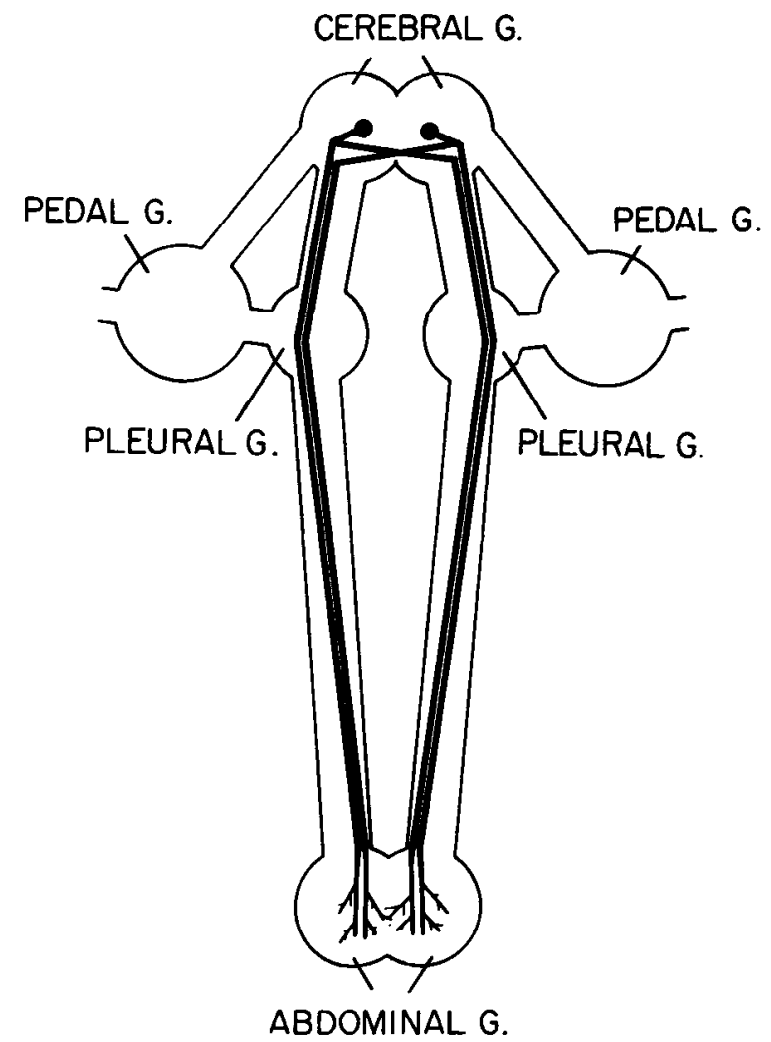

Figure 4. Proposed axonal projections of the CB1 neurons, based on retrograde labeling from the abdominal ganglion (Hawkins, 1989), antidromic stimulation by shocking the pleurat-abdominal connectives, intracellular dye filling (Fig. 3), and 5-HT immunofluorescence (Fig. 3 and Longley and Longley, 1986). The diagram is modified from Longley and Longley (1986).

mM: $260 \mathrm{NaCl}, 10 \mathrm{KCl}, 60 \mathrm{CaCl}_{2}, 140 \mathrm{MgCl}_{2}$ ) in order to raise action potential threshold and thereby reduce interneuron firing. This treatment produced no decrease in the duration or magnitude of facilitation. While these results by no means exclude the possibility that the facilitation observed during CB1 stimulation may be via other interneurons, the results are consistent with the idea that CB1 produces facilitation directly.

The facilitation produced by $\mathrm{CB} 1$ stimulation could be either pre- or postsynaptic. Possible postsynaptic mechanisms include hyperpolarization of the membrane potential (increasing the driving force) and increased input impedance. LFS cells showed either no change in membrane potential or occasionally a slight depolarization following CB1 stimulation. In order to test whether CB1 stimulation produces changes in LFS input impedance, at the end of 6 out of 10 EPSP facilitation experiments, the LFS cell was injected with hyperpolarizing pulses lasting 600-800 msec. The current was adjusted to produce a membrane change similar in magnitude to that of the EPSP. Using the same test protocol as in the PSP experiments, hyperpolarizing pulses were injected at 30 -sec intervals before, during, and

Figure 3. Serotonin immunoreactivity in a CB1 neuron. The cell was identified electrophysiologically and injected with Lucifer yellow, and then the ganglion was processed for wholemount 5-HT immunocytochemistry. $A_{i}$, The region of the right B cluster viewed with Lucifer yellow filters, showing the injected CB1 cell (arrow). Two other cells that could be distinguished by their relative positions were also injected. $A_{2}$, The same region viewed with rhodamine filters, showing 5-HT immunofluorescence in the CB1 neuron (arrow). The other neurons which had been injected with Lucifer yellow, do not show 5-HT immunofluorescence, indicating that the fluorescence is not due to Lucifer yellow "breakthrough" of the filters. $B$, A similar pair of photographs of the axon of the injected cell in the left cerebral-pleural connective. 

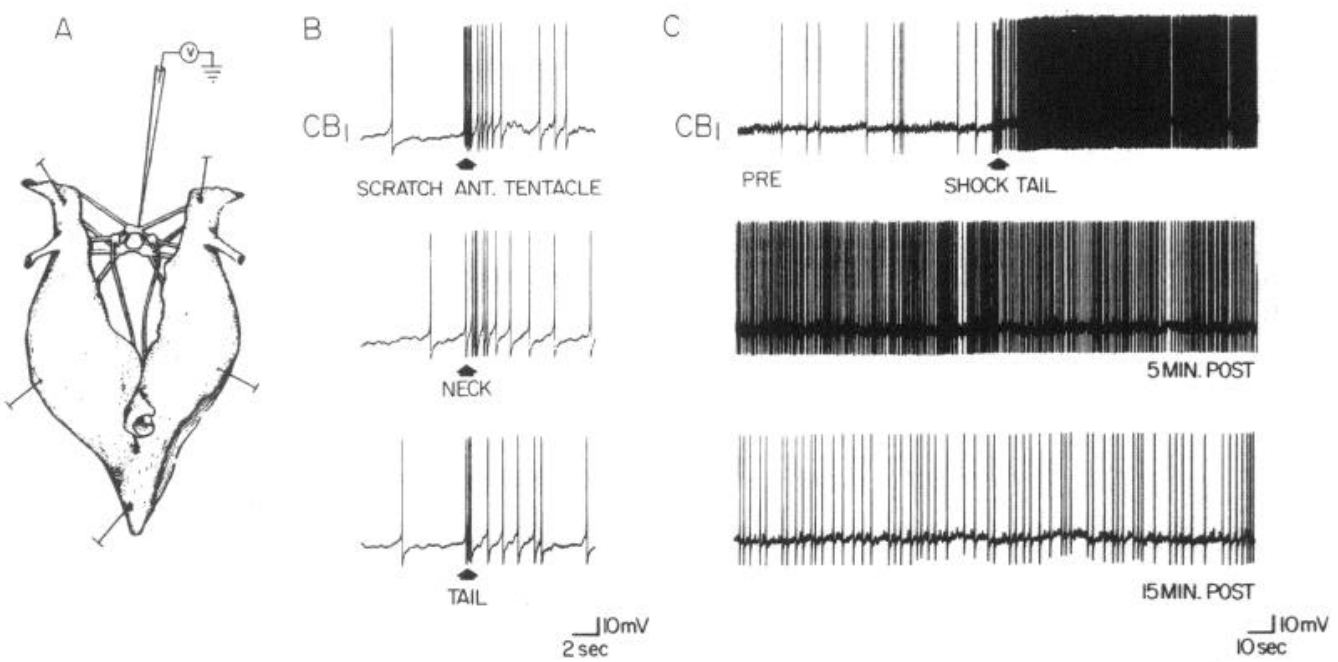

Figure 5. Response of $\mathrm{CB1}$ to cutaneous stimulation. $A$, Drawing of the semiintact preparation used for recording from CB1 while stimulating the skin. $B$, Examples of the response of a CB1 neuron to moderate intensity stimulation (scratch) delivered to the anterior tentacles, neck, and tail. $C$, Example of the response of the same $C B 1$ neuron to shocking the tail $(75 \mathrm{~mA}$ a.c., $1.0 \mathrm{sec})$. The first 2 traces are continuous, showing firing of the CB1 neuron before (Pre) and during the first $5 \mathrm{~min}$ after (Post) tail shock. The third trace shows the firing rate of the neuron 15 min after tail shock. The neuron recorded from in this experiment was the same one that is shown in Figure 2.

after CB1 stimulation. There was no change in the magnitude of the hyperpolarization produced by the current pulses during CB1 stimulation $\left(\mathrm{F}_{1.40}=0.64\right.$, not significant $)$.

\section{Stimulation of $C B 1$ produces spike broadening in LE sensory neurons}

The results of the above experiments show that postsynaptic changes in membrane potential or input resistance (as measured in the cell body) cannot account for the EPSP facilitation during and following CB1 stimulation. Although we have not excluded other postsynaptic mechanisms (for example, changes in receptor sensitivity), our results are consistent with the possibility that EPSP facilitation is mediated at least in part presynaptically. Application of 5-HT has previously been shown to cause broadening of the action potential in LE sensory neurons, contributing to presynaptic facilitation. This effect is more dramatic in seawater containing TEA, which blocks a non-5-HT-sensitive $\mathrm{K}^{+}$ current, making the action potential more sensitive to changes in the remaining currents (Klein and Kandel, 1978). We were interested to see whether stimulation of $\mathrm{CB} 1$ also produces spike broadening in LE cells. Using an isolated ring and abdominal ganglion preparation, we identified CB1 neurons as described above. Next, we impaled LE sensory cells and perfused the ganglia with seawater containing $100 \mathrm{~mm}$ TEA. Action potentials were produced in the LE cells by $5 \mathrm{msec}$, depolarizing pulses with a 20 -sec intertrial interval. After at least 3 pretests, the CB1 cell was fired at $1-3 \mathrm{~Hz}$ for $60-200 \mathrm{sec}$. LE cells were stimulated for 3-10 trials during CB1 stimulation and 3 trials poststimulation.

An example of the results is shown in Figure $7 A$, and the average of 10 experiments is plotted in Figure $7 \mathrm{~B}$. Spike width has been normalized to the last pretest $(-1)$. On average there was a small decrease in spike width from trial -3 to -1 . Following the start of $\mathrm{CB} 1$ stimulation, the spike width increased to $133 \pm 19 \%$ of pretest by trial 3 . After the end of CB1 stimulation, the spike width declined and was $117 \pm 11 \%$ of pretest by posttest 3 . The average spike width during and following CB1 stimulation was significantly greater than pretest $\left(\mathrm{F}_{1,45}=\right.$ 50.16, $p<0.01$ ), and planned comparisons at the individual time points show significant facilitation on trial 3 during $C B 1$ stimulation and posttests 1 and 2 ( $p<0.01$ in each case). These results are similar in time course and magnitude to the results of the PSP facilitation experiments. In addition, the magnitude of spike broadening, while modest, is similar to that seen following tail stimulation (Mackey et al., 1987).

\section{Discussion}

\section{Identified serotonergic facilitator neurons}

In summary, we have identified a pair of neurons in the cerebral ganglia (the left and right CB1 cells) which show both 5-HT histofluorescence and immunoreactivity. Each CB1 sends bilateral axons into the pleural-abdominal connectives, which are probably the 5-HT immunoreactive axons "S1" and "S2" that Longley and Longley (1986) showed project to the abdominal ganglion. The CB1 cells are excited by cutaneous stimuli known to produce dishabituation and sensitization of the gill- and siphon-withdrawal reflex and facilitation of the synaptic connections of the LE sensory neurons. Intracellular stimulation of the CB1 cells at a frequency similar to their response to cutaneous stimuli produces facilitation of the EPSPs from the LE cells to follower cells. The facilitation is not reduced in a high-divalent solution that raises the firing threshold of interneurons, suggesting the effect is direct. The EPSP facilitation cannot be explained by postsynaptic changes in membrane potential or conductance. Rather, intracellular stimulation of $\mathrm{CB} 1$ produces broadening of the sensory neuron action potential in TEA, suggesting that the facilitation is at least in part presynaptic.

Although the evidence that the CB1 neurons are serotonergic is strong, it is not complete. 5-HT mimics the physiological effects of $\mathrm{CB} 1$, and 2 independent histochemical techniques have shown that $\mathrm{CB} 1$ contains 5-HT. However, it would be desirable to use biochemical techniques to test whether CB1 also synthesizes and releases 5-HT.

A second issue is whether the facilitatory effects of $\mathrm{CB} 1$ are direct. Although suggestive, experiments conducted in high divalents by no means exclude the possibility that at least part of the effect may be mediated by interposed interneurons. Preliminary evidence indicates that $\mathrm{CB} 1$ has no synaptic connections 
A
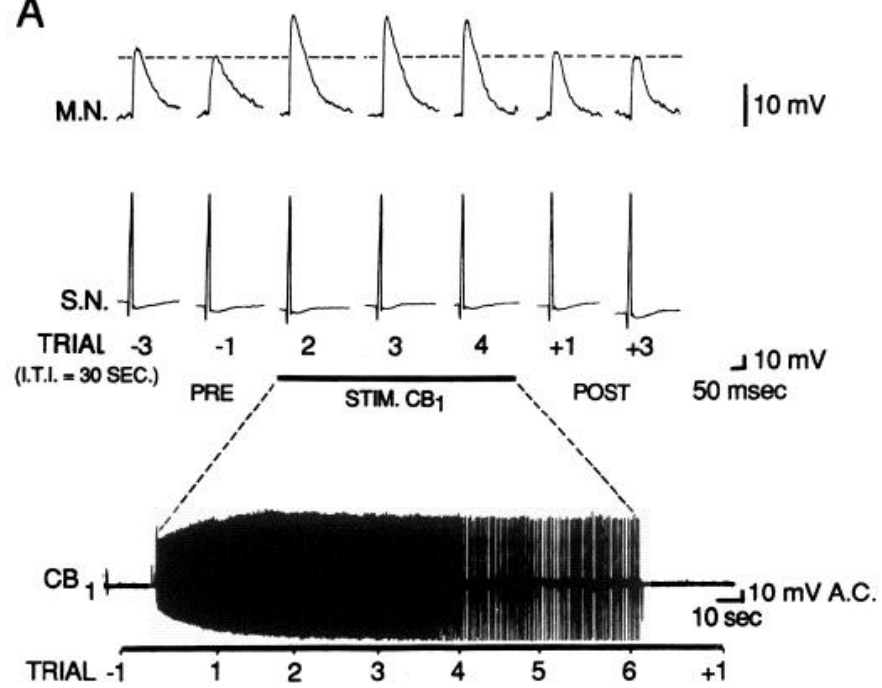

B

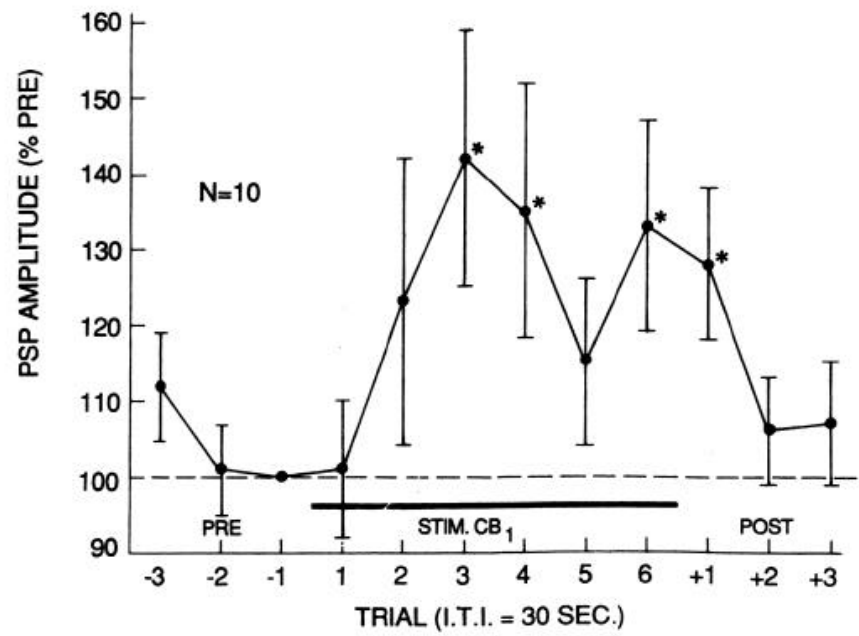

Figure 6. Intracellular stimulation of $\mathrm{CB} 1$ produces facilitation of the EPSP from a siphon sensory neuron to a motor neuron. $A$, Example of facilitation. The sensory neuron $(S . N$.) was fired intracellularly, producing an EPSP in the motor neuron (M.N.) with an intertrial interval (I.T.I.) of $30 \mathrm{sec}$. There were at least 3 trials before (Pre), 6 trials during, and 3 trials after (Post) intracellular stimulation of $\mathrm{CB} 1$, which is shown on a different time scale in the third trace. $B$, Average facilitation in 10 experiments. The amplitude of the EPSP has been normalized to the value on the last pretest $($ Trial -1$)$. Error bars = standard errors of the means. ${ }^{*}=$ significant facilitation compared to pretest $(p<0.01)$.

with the abdominal facilitatory neuron L29. We have not tested whether there are connections with the facilitatory neurons L22 or L28. It will also be important to test whether at least some of the serotonergic varicosities observed in the region of the LE sensory neurons are contributed by CB1.

The facilitation produced by stimulating a single $\mathrm{CB} 1$, although reliable, is modest. We have not yet tested whether simultaneously stimulating both $\mathrm{CB} 1$ neurons enhances the facilitatory effect. We doubt, however, that the $2 \mathrm{CB} 1$ cells can account for most of the facilitation produced by tail shock. First, tail or nerve stimulation recruits not only CB1 but also the abdominal interneurons L22, L28, and L29 (Hawkins et al., 1981; Hawkins and Schacher, 1989). Each of these neurons has
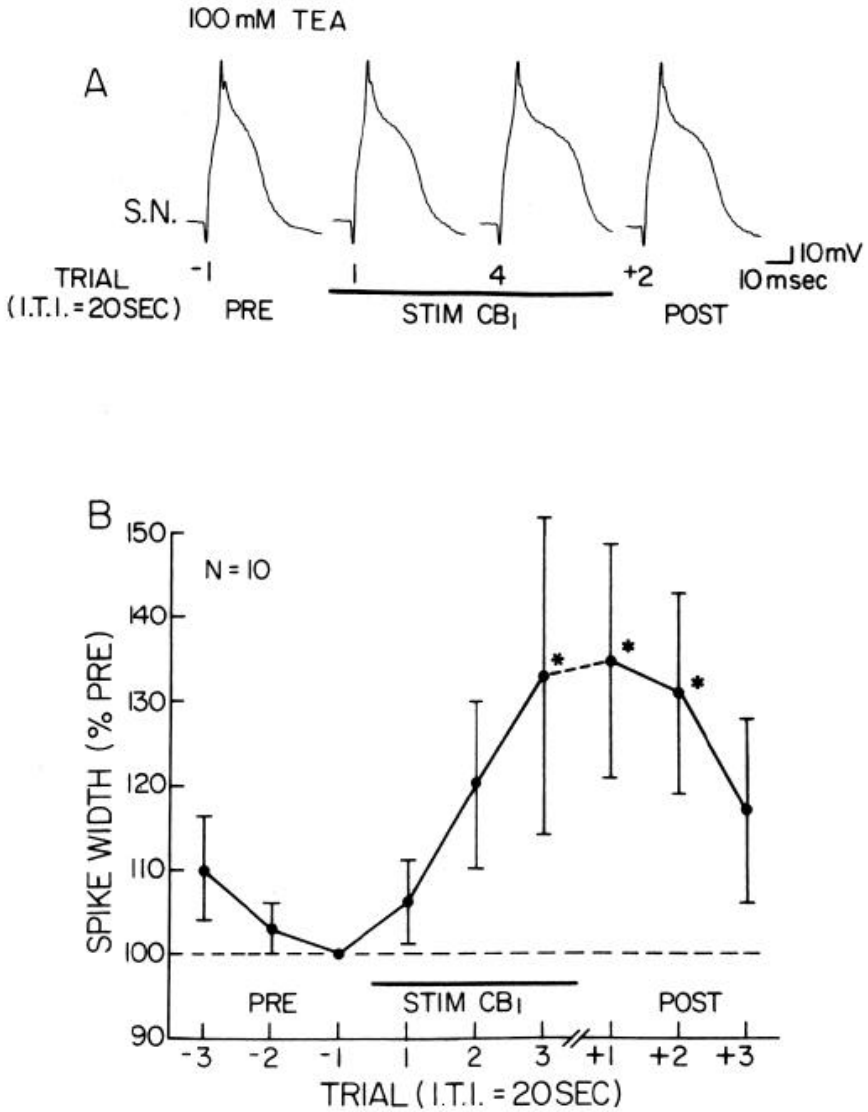

Figure 7. Intracellular stimulation of $\mathrm{CB} 1$ produces broadening of the action potential in a siphon sensory neuron in seawater containing 100 mM TEA. A, Example of spike broadening. The intertrial interval (I.T.I.) in these experiments was $20 \mathrm{sec}$. $B$, Average spike broadening in 10 experiments. The duration of the action potential has been normalized to the value on the last pretest (Trial -1$)$. Only the first 3 trials during CB1 stimulation are plotted.

been shown to produce facilitation comparable to that produced by CB1 (Hawkins et al., 1981). Second, in experiments in which a single $\mathrm{CB} 1$ has been hyperpolarized to prevent spiking, tail stimulation is still capable of producing facilitation. Further experiments will be needed to determine whether there are quantitative differences in either the magnitude or time course of facilitation under conditions in which CB1 is prevented from spiking. Third, since cutaneous stimuli such as tail shock recruit both facilitation and inhibition of the siphon sensory neurons (Mackey et al., 1987), the actual facilitation elicited by tail shock is probably greater than the net facilitation observed. Therefore, comparing the facilitation produced by firing $\mathrm{CB} 1$ to the net facilitation measured following tail shock probably overestimates the contribution of the CB1 cells to total facilitation. For these reasons, we think that there may be additional facilitator neurons for the gill- and siphon-withdrawal reflex, some of which may be serotonergic (Glanzman et al., 1989). If so, it will be interesting to see whether their properties are similar to or different from those of CB1.

\section{Comparison with other identified facilitator neurons}

Hawkins et al. (1981) reported the identification of 3 groups of neurons on the ventral surface of the left abdominal ganglion which produce heterosynaptic facilitation of complex PSPs in 
the motor neuron L7: L22 (a cluster of about 3 cells), L28 (a single cell), and L29 (a cluster of about 5 weakly electrically coupled cells). All 3 types of cells are excitatory interneurons in the circuit for the gill- and siphon-withdrawal reflex. The L29 cells have been the most thoroughly studied. Brief, high-frequency intracellular stimulation of an L29 cell produces facilitation of monosynaptic EPSPs from siphon sensory cells to follower cells and broadening of action potentials in sensory cells in TEA solution (Hawkins et al., 1981; Hawkins, 1981). The facilitation produced by L 22 and L28 has not been analyzed and may involve other mechanisms, such as PTP of the L22L7 connection (Hawkins et al., 1981). Although indirect evidence initially suggested that the L29 cells might be serotonergic (Bailey et al., 1981, 1983), no serotonergic neurons have been seen on the left ventral surface of the abdominal ganglion with either histofluorescence or immunocytochemistry (Tritt et al., 1983; Goldstein et al., 1984; Ono and McCaman, 1984; Longley and Longley, 1986; Salimova et al., 1987). Moreover, identified L29 cells marked with fluorescent dye do not show 5-HT histofluorescence or immunofluorescence (Kistler et al., 1985; Hawkins, 1989).

It is interesting to speculate why Aplysia has several different populations of facilitator neurons for the gill- and siphon-withdrawal reflex. A comparison of L29 and CB1 may provide insights into different roles subserved by these interneurons. L29 and $C B 1$ show significant differences in several characteristics including (1) receptive field, (2) response to graded stimuli, and (3) time course of facilitation. In terms of receptive field, L29 shows some degree of site specificity. It receives excitatory input from the posterior part of the body, but it is inhibited by stimuli directed to the anterior part of the body (Hawkins and Schacher, 1989). CB1, by contrast, is excited by tactile stimulation over all areas of the body including the tail, siphon, mantle, neck, rhinophores, and anterior tentacles. Stimulation of any of these areas can produce dishabituation and sensitization of the gilland siphon-withdrawal reflex (Pinsker et al., 1970; Carew et al., 1971, 1981; Mackey et al., 1988; Marcus et al., 1988; V. F. Castellucci and R. D. Hawkins, unpublished observations). L29 thus appears to play a more local role in the gill- and siphonwithdrawal reflex, whereas CB1 may have a more general role. For example, Hawkins et al. (1981) have suggested that 1 function of L29 may be to modulate the time course of habituation of the withdrawal reflex to different intensity stimuli. The fact that L29 is an excitatory interneuron in the withdrawal circuit, with direct and indirect connections to motor neurons, may also explain why it has a limited receptive field. CB1, on the other hand, does not appear to be an excitatory interneuron and does not show site specificity.

L29 and CB1 also differ in terms of their response to graded tactile stimuli. L29 neurons are sensitive to light tactile stimuli, responding with a high-frequency burst of spikes. Noxious stimuli prolong the spiking to the duration of the stimuli, but not beyond (Hawkins and Schacher, 1989). CB1 neurons respond to moderate tactile stimuli with a brief phasic response and respond to noxious stimuli with a tonic increase in firing lasting many minutes. These differences in response characteristics may be related to the differences in time course of facilitation produced by these neurons. Brief intracellular stimulation of L29 produces facilitation that peaks within $10 \mathrm{sec}$ and subsequently declines slowly with a time course parallel to the decrement seen prior to stimulation (Hawkins et al., 1981). The facilitation produced by prolonged CB1 stimulation peaks after $90 \mathrm{sec}$ and remains elevated for the duration of $\mathrm{CB} 1$ firing. Thereafter, it returns to near baseline within $1 \mathrm{~min}$. These results suggest that L29 and CB1 might play different roles in dishabituation and sensitization of the withdrawal reflex. Marcus et al. (1988) showed that dishabituation can be produced by relatively weak stimulation and has a rapid onset, whereas sensitization requires stronger stimulation and may have a delayed onset (this distinction between dishabituation and sensitization is also consistent with the results of Pinsker et al., 1970; Carew et al., 1971, 1981; Mackey et al., 1988; Castellucci and Hawkins, unpublished observations). The characteristics of L29 (low threshold and rapid facilitation) suggest that it may be preferentially involved in dishabituation, whereas the characteristics of CB1 (higher threshold and slower facilitation) suggest that it may be preferentially involved in sensitization. However, there is considerable overlap, and both neurons could contribute to both effects.

\section{Serotonergic systems in Aplysia}

There are now several identified serotonergic neurons in Aplysia, permitting a comparison of serotonergic function in different systems within the same organism. The MCCs modulate the feeding circuit at several sites, increasing the rhythmic activity of the feeding pattern generator, depolarizing buccal motor neurons, and enhancing buccal muscle contractions (Weiss et al., 1982). $R B_{\mathrm{HE}}$ accelerates heart rate and thereby influences circulation (Mayeri et al., 1974; Liebeswar et al., 1975). CB1 produces facilitation of LE sensory cells involved in defensive withdrawal. In all 3 cases the cellular responses are characterized by slow and predominantly modulatory effects which are mediated, at least in part, by cAMP (Brunelli et al., 1976; Mandelbaum et al., 1979; Bernier et al., 1982; Weiss et al., 1982).

We have not yet examined whether CB1, like MCC, may have modulatory effects at multiple sites within the siphonwithdrawal circuit. Our data show no evidence of conventional synaptic input to either L7 or LFS motor neurons. It will be interesting to test whether firing CB1 mimics the effects of 5-HT on neuronal elements in the circuit in other ways, such as inhibiting the L30 inhibitory interneurons or increasing the spontaneous firing rate of the LFS siphon motor neurons (Frost et al., 1988). We have also not yet tested whether CB1's role is limited to facilitation of the EPSP from siphon sensory neurons to gill and siphon motor neurons or generalizes to sensory neurons in other response systems. Preliminary evidence suggests that CB1 has little or no effect on pleural sensory neurons that mediate the tail withdrawal reflex. Possible modulatory effects on cerebral sensory neurons have not yet been examined. Since some of the cerebral sensory neurons respond to 5-HT and nerve stimulation with spike broadening, and others respond with spike narrowing (Rosen et al., 1989), it will be interesting to see whether firing CB1 can mimic either of these effects. The identification of the CB1 neurons should allow us to begin to answer some of these questions and hopefully thereby to obtain new insights into the organization of serotonergic systems in Aplysia.

\section{References}

Abrams, T. W., V. F. Castellucci, J. S. Camardo, E. R. Kandel, and P. E. Lloyd (1984) Two endogenous neuropeptides modulate the gill and siphon withdrawal reflex in Aplysia by presynaptic facilitation involving cAMP-dependent closure of a serotonin-sensitive potassium channel. Proc. Natl. Acad. Sci. USA 81: 7956-7960.

Bailey, C. H., R. D. Hawkins, M. C. Chen, and E. R. Kandel (1981) Interneurons involved in mediation and modulation of gill-with- 
drawal reflex in Aplysia. IV. Morphologial basis of presynaptic facilitation. J. Neurophysiol. 45: 340-360.

Bailey, C. H., R. D. Hawkins, and M. Chen (1983) Uptake of (3H) serotonin in the abdominal ganglion of Aplysia californica: Further studies on the morphological and biochemical basis of presynaptic facilitation. Brain Res. 272: 71-81.

Bernier, L., V. F. Castellucci, E. R. Kandel, and J. H. Schwartz. (1982) Facilitatory transmitter causes a selective and prolonged increase in adenosine $3^{\prime} 5^{\prime}$-monophosphate in sensory neurons mediating the gill and siphon withdrawal reflex in Aplysia. J. Neurosci. 2: 1682-1691.

Brunelli, M., V. Castellucci, and E. R. Kandel (1976) Synaptic facilitation and behavioral sensitization in Aplysia: Possible role of serotonin and cAMP. Science 194: 1178-1181.

Carew, T. J., V. F. Castellucci, and E. R. Kandel (1971) An analysis of dishabituation and sensitization of the gill-withdrawal reflex in Aplysia. Int. J. Neurosci. 2: 79-98.

Carew, T. J., E. T. Walters, and E. R. Kandel (1981) Classical conditioning in a simple withdrawal reflex in Aplysia californica. J. Neurosci. 1: 1426-1437.

Frost, W. N., G. A. Clark, and E. R. Kandel (1988) Parallel processing of short-term memory for sensitization in Aplysia. J. Neurobiol. 19: 297-334.

Glanzman, D. L., S. L. Mackey, R. D. Hawkins, A. Dyke, P. E. Lloyd, and E. R. Kandel (1989) Depletion of serotonin in the nervous system of Aplysia reduces the behavioral enhancement of gill withdrawal as well as the heterosynaptic facilitation produced by tail shock. J. Neurosci. 9: 4200-4213.

Goldstein, R., H. B. Kistler, H. W. M. Steinbusch, and J. H. Schwartz (1984) Distribution of serotonin-immunoreactivity in juvenile Aplysia. Neuroscience 11: 535-547.

Hawkins, R. D. (1981) Interneurons involved in mediation and modulation of gill-withdrawal reflex in Aplysia. III. Identified facilitating neurons increase $\mathrm{Ca}^{2+}$ current in sensory neurons. J. Neurophysiol. 45: 327-339.

Hawkins, R. D. (1989) Localization of potential serotonergic facilitator neurons in Aplysia by glyoxylic acid histofluorescence combined with retrograde fluorescent labeling. J. Neurosci. 9: 4214-4226.

Hawkins, R. D., and S. Schacher (1989) Identified facilitator neurons L29 and L28 are excited by cutaneous stimuli used in dishabituation, sensitization, and classical conditioning of Aplysia. J. Neurosci. 9: $4236-4245$.

Hawkins, R. D., V. F. Castellucci, and E. R. Kandel (1981) Interneurons involved in mediation and modulation of gill-withdrawal reflex in Aplysia. II. Identified neurons produce heterosynaptic facilitation contributing to behavioral sensitization. J. Neurophysiol. 45: 315326.

Hening, W. A., E. T. Walters, T. J. Carew, and E. R. Kandel (1979) Motorneuronal control of locomotion in Aplysia. Brain Res. 179:231253.

Jahan-Parwar, B., and S. M. Fredman (1976) Cerebral ganglion of Aplysia: Cellular organization and origin of nerves. Comp. Biochem. Physiol. 54A: 347-357.

Kistler, H. B., Jr., R. D. Hawkins, J. Koester, H. W. M. Steinbusch, E. R. Kandel, and J. H. Schwartz (1985) Distribution of serotonin- immunoreactive cell bodies and processes in the abdominal ganglion of mature Aplysia. J: Neurosci. 5: 72-80.

Klein, M., and E. R. Kandel (1978) Presynaptic modulation of voltagedependent $\mathrm{Ca}^{2+}$ current: Mechanism for behavioral sensitization. Proc. Natl. Acad. Sci. USA 75: 3512-3516.

Liebeswar, G., J. E. Goldman, J. Koester, and E. Mayeri (1975) Neural control of circulation in Aplysia. III. Neurotransmitters. J. Neurophysiol. 38: 767-779.

Longley, R. D., and A. J. Longley (1986) Serotonin immunoreactivity of neurons in the gastropod Aplysia californica. J. Neurobiol. 17:339358.

Mackey, S. L., E. R. Kandel, and R. D. Hawkins (1986) Neurons in 5-HT containing region of cerebral ganglia produce facilitation of LE cells in Aplysia. Soc. Neurosci. Abstr. 12: 1340.

Mackey, S. L., D. L. Glanzman, S. A. Small, A. M. Dyke, E. R. Kandel and R. D. Hawkins (1987) Tail shock produces inhibition as well as sensitization of the siphon-withdrawal reflex of Aplysia: Possible behavioral role for presynaptic inhibition mediated by the peptide Phe-Met-Arg-Phe-NH . Proc. Natl. Acad. Sci. USA 84: 8730-8734.

Mackey, S. L., N. Lalevic, R. D. Hawkins, and E. R. Kandel (1988) Comparison of dishabituation and sensitization of the gill-withdrawal reflex in Aplysia. Soc. Neurosci. Abstr. 14: 842.

Mandelbaum, D. E., J. Koester, M. Schonberg, and K. R. Weiss (1979) Cyclic AMP mediation of the excitatory effect of serotonin in the heart of Aplysia. Brain Res. 177: 388-394.

Marcus, E. A., T. G. Nolen, C. H. Rankin, and T. J. Carew (1988) Behavioral dissociation of dishabituation, sensitization, and inhibition in Aplysia. Science 241: 210-213.

Mayeri, E., J. Koester, I. Kupfermann, G. Liebeswar, and E. R. Kandel (1974) Neural control of circulation in Aplysia. I. Motoneurons. J. Neurophysiol. 37: 458-475.

Ono, J., and R. E. McCaman (1984) Immunocytochemical localization and direct assays of serotonin-containing neurons in Aplysia. Neuroscience $11: 549-560$.

Pinsker, H., I. Kupfermann, V. Castellucci, and E. R. Kandel (1970) Habituation and dishabituation of the gill-withdrawal reflex in $A p l y$ sia. Science 167: 1740-1742.

Rosen, S. C., A. J. Susswein, E. C. Cropper, K. R. Weiss, and I. Kupfermann (1989) Selective modulation of spike duration by serotonin and the neuropeptides FMRFamide, $\mathrm{SCP}_{\mathrm{B}}$, buccalin, and myomodulin in different classes of mechanoafferent neurons in the cerebral ganglion of Aplysia. J. Neurosci. 9: 390-402.

Salimova, N. B., D. A. Sakharov, I. Milosevic, T. M. Turpaev, and L. Rakic (1987) Monoamine-containing neurons in the Aplysia brain. Brain Res. 400: 285-299.

Tritt, S. H., I. P. Lowe, and J. H. Byrne (1983) A modification of the glyoxylic acid induced histofluorescence technique for demonstration of catecholamines and serotonin in tissues of Aplysia californica. Brain Res. 259: 159-162.

Weiss, K. R., U. T. Koch, J. Koester, S. C. Rosen, and I. Kupfermann (1982) The role of arousal in modulating feeding behavior of Aplysia: Neural and behavioral studies. In The Neural Basis of Feeding and Reward, B. G. Hoebel and D. Norin, eds., Haer Institute, Brunswick, ME. 